\title{
Long-term safety and feasibility of three-vessel multimodality intravascular imaging in patients with ST-elevation myocardial infarction: the IBIS-4 (integrated biomarker and imaging study) substudy
}

\author{
Masanori Taniwaki • Maria D. Radu • Hector M. Garcia-Garcia • \\ Dik Heg • Henning Kelbæk • Lene Holmvang • Aris Moschovitis • \\ Stephane Noble · Giovanni Pedrazzini $\cdot$ Kari Saunamäki · Jouke Dijkstra • \\ Ulf Landmesser - Peter Wenaweser · Bernhard Meier - Giulio G. Stefanini • \\ Marco Roffi - Thomas F. Lüscher · Stephan Windecker · Lorenz Räber
}

Received: 24 November 2014 / Accepted: 22 February 2015/Published online: 28 February 2015

(C) Springer Science+Business Media Dordrecht 2015

\begin{abstract}
We assessed the feasibility and the procedural and long-term safety of intracoronary (i.c) imaging for documentary purposes with optical coherence tomography (OCT) and intravascular ultrasound (IVUS) in patients with acute ST-elevation myocardial infarction (STEMI) undergoing primary PCI in the setting of IBIS-4 study. IBIS4 (NCT00962416) is a prospective cohort study conducted at five European centers including 103 STEMI patients who underwent serial three-vessel coronary imaging during primary PCI and at 13 months. The feasibility parameter was successful imaging, defined as the number of pullbacks suitable for analysis. Safety parameters included the frequency of peri-procedural complications, and major
\end{abstract}

The IBIS4 trial was supported by the Swiss National Science Foundation, and is registered at:

http://www.clinicaltrials.gov/ct2/show/NCT00617084.

Electronic supplementary material The online version of this article (doi:10.1007/s10554-015-0631-0) contains supplementary material, which is available to authorized users.

M. Taniwaki - A. Moschovitis - P. Wenaweser - B. Meier .

G. G. Stefanini · S. Windecker · L. Räber ( $\square)$

Department of Cardiology, Swiss Cardiovascular Center Bern,

Bern University Hospital, 3010 Bern, Switzerland

e-mail: lorenz.raeber@insel.ch

M. D. Radu · H. Kelbæk · L. Holmvang · K. Saunamäki

Rigshospitalet, Copenhagen, Denmark

H. M. Garcia-Garcia

Erasmus MC, Rotterdam, The Netherlands

D. Heg

Clinical Trial Unit, University of Bern, Bern, Switzerland adverse cardiac events (MACE), a composite of cardiac death, myocardial infarction (MI) and any clinically-indicated revascularization at 2 years. Clinical outcomes were compared with the results from a cohort of 485 STEMI patients undergoing primary PCI without additional imaging. Imaging of the infarct-related artery at baseline (and follow-up) was successful in $92.2 \%(96.6 \%)$ of patients using OCT and in $93.2 \%$ (95.5\%) using IVUS. Imaging of the non-infarct-related vessels was successful in $88.7 \%$ (95.6\%) using OCT and in $90.5 \%(93.3 \%)$ using IVUS. Periprocedural complications occurred $<2.0 \%$ of OCT and none during IVUS. There were no differences throughout 2 years between the imaging and control group in terms of MACE (16.7 vs. $13.3 \%$, adjusted HR1.40, $95 \%$ CI $0.77-2.52, \quad p=0.27)$. Multi-modality three-vessel i.c. imaging in STEMI patients undergoing primary PCI is consistent a high degree of success and can be performed safely without impact on cardiovascular events at longterm follow-up.

\author{
S. Noble $\cdot$ M. Roffi \\ University Hospital, Geneva, Switzerland \\ G. Pedrazzini \\ Cardiocentro, Lugano, Switzerland \\ J. Dijkstra \\ Leiden University Medical Center, Leiden, Netherlands \\ U. Landmesser . T. F. Lüscher \\ University Heart Center, Cardiology, University Hospital Zurich, \\ Zurich, Switzerland
}


Keywords Optical coherence tomography · Intravascular ultrasound - ST-segment elevation myocardial infarction . Long-term safety

$\begin{array}{ll}\text { Abbreviations and acronyms } \\ \text { ARC } & \text { Academic research consortium } \\ \text { CI } & \text { Confidence interval } \\ \text { DES } & \text { Drug-eluting stent } \\ \text { MACE } & \text { Major adverse cardiac event } \\ \text { MI } & \text { Myocardial infarction } \\ \text { ST } & \text { Stent thrombosis } \\ \text { TLF } & \text { Target lesion failure } \\ \text { TLR } & \text { Target lesion revascularization } \\ \text { IVUS } & \text { Intravascular ultrasound } \\ \text { IVUS-VH } & \text { IVUS virtual histology } \\ \text { OCT } & \text { Optical coherence tomography } \\ \text { PCI } & \text { Percutaneous coronary intervention } \\ \text { STEMI } & \text { ST-elevation myocardial infarction }\end{array}$

\section{Introduction}

Multimodality intracoronary (i.c.) imaging using optical coherence tomography (OCT), grayscale intravascular ultrasound (IVUS), and IVUS-virtual histology (IVUS-VH) provides means to characterize coronary atherosclerosis and optimize stent implantation in a comprehensive manner. Based on near-infrared light, OCT visualizes in great detail the vascular luminal integrity, thrombus, and characteristics of plaque vulnerability and facilitates the detection of geographical miss, stent strut malapposition and edge dissections following stent implantation [1]. Although the resolution of IVUS is an order of magnitude lower than OCT, the technology provides a greater penetration depth and therefore the ability to assess plaque volume [2]. IVUS-VH is based on radiofrequency analysis and has been proposed as a method to assist the evaluation of plaque composition, particularly necrotic core [3]. The combined use of these sound- and light-based modalities theoretically increases the accuracy to detect "vulnerable" plaques $[4,5]$, and may thus provide a complete platform to exploit future treatment targets. Additionally, these technologies have the potential to improve cardiovascular outcomes by optimizing percutaneous coronary intervention (PCI), particularly in STEMI patients [6].

Nevertheless, the use of intracoronary imaging catheters may be accompanied by procedural complications including injury of the endothelium, which may increase the risk of short- and long-term events. Despite an increasing use of intracoronary imaging in daily practice, there is insufficient data on the feasibility and safety of multimodality imaging.
We therefore aimed to analyse the feasibility and short- and long-term safety of patients enrolled in the IBIS-4 imaging study, a prospective, observational multicenter study with the objective to assess the arterial healing after biolimuseluting stent implantation versus bare metal stent implantation and to investigate the changes in atherosclerosis on the background of a high intensity statin therapy in the two non-infarct related arteries. [7].

\section{Methods}

Study population

IBIS 4 (NCT00962416) is a prospective cohort study nested into the COMFORTABLE-AMI, a trial comparing the safety and efficacy of a biolimus-eluting stent with a bare metal stent in 1161 STEMI patients undergoing primary PCI at 11 international centers [8]. A total of 103 patients were enrolled in this imaging study using OCT, IVUS and IVUS-VH to assess the arterial healing response following stent implantation and to quantify changes in atherosclerotic plaque characteristics in non-infarct related arteries in the presence of high dose statin therapy (rosuvastatin $40 \mathrm{mg}$ daily).

Patients enrolled in the COMFORTABLE-AMI trial were eligible for participating in the IBIS 4 study when the following criteria were met: age $<90$ years, haemodynamic stability allowing the administration of nitroglycerine, preserved renal function (GFR $>30 \mathrm{ml} / \mathrm{min}$ ), $\geq$ TIMI II flow of the infarct-related artery at the end of the intervention, and a coronary anatomy considered suitable for intracoronary imaging (e.g. absence of vessel tortuosity).

Following PCI, multimodality imaging with OCT followed by IVUS/IVUS-VH was performed, first addressing the treated culprit lesion, and then the two non-infarct related arteries. Care was taken to maintain an activated clotting time $>250 \mathrm{~s}$, and prior to each pullback, i.c. nitroglycerine $(200 \mu \mathrm{g})$ was administered to achieve maximal vasodilatation. After complete acquisition, imaging pullbacks were analyzed offline by an independent CoreLab (Cardialysis B.V., Rotterdam). The study protocol defined the intracoronary imaging as documentary. Although intervening physicians were not blinded to the acquired pullbacks, they were recommended not to perform any corrective measures based on the imaging findings.

All patients were scheduled for repeat angiography and the same three vessel multimodality imaging protocol at 13 months. In total, they were followed up to 2 years, and events were recorded at 30 days, 1 year, and 2 years. Per protocol, all patients received rosuvastatin $40 \mathrm{mg}$ once daily throughout the 13 month follow-up period.

The control group of the present study consisted of 485 patients who were simultaneously enrolled in the 
COMFORTABLE-AMI study at the five imaging centers, but who did not undergo imaging although they formally fulfilled the inclusion criteria for the imaging sub-study. All patients provided written informed consent for the participation in both the COMFORTABLE-AMI study and IBIS 4.

\section{OCT}

OCT images were acquired using the frequency domain (FD) C7 console system (Lightlab, St. Jude, Westford, MA, USA) and the Dragonfly catheter at a pullback speed of $20 \mathrm{~mm} / \mathrm{sec}$. Blood clearance was achieved using the nonobstructive technique with a power injector applying flushing rates of 3-6 $\mathrm{ml} / \mathrm{sec}$ depending on vessel size. The regions of interest included in the culprit vessel: at least $5 \mathrm{~mm}$ of the proximal and distal reference vessel segments; and as much as possible of the proximal non-infarct-related arteries, though a minimum of $40 \mathrm{~mm}$ as measured from the respective ostia.

\section{IVUS}

IVUS-images were acquired with the Volcano s5 system (Volcano Corp., Rancho Cordova, CA) and the 2.9 French Eagle Eye catheter, which uses a $20 \mathrm{MHz}$ transducer, at a continuous motorized pullback rate of $0.5 \mathrm{~mm} / \mathrm{sec}(R-100$ pullback device; Volcano Therapeutics). The regions of interest were the same as for OCT, as were the storage of pullbacks and transfer to CoreLab for further offline analysis.

\section{Antithrombotic therapy regimen}

All patients were loaded with aspirin $(\geq 250 \mathrm{mg}$ ) prior to the procedure. In centers where prasugrel was available, an initial dose of $60 \mathrm{mg}$ (including patients preloaded with clopidogrel) was given followed by a daily maintenance dose of $10 \mathrm{mg}$. If prasugrel was not available or contraindicated, clopidogrel was administered at a loading dose of $600 \mathrm{mg}$, followed by $75 \mathrm{mg}$ twice daily for 7 days, followed by a maintenance dose of $75 \mathrm{mg}$ daily. Dual antiplatelet therapy was prescribed for the duration of 13 months.

\section{Feasibility parameters}

Feasibility was defined as the acquisition of a pullback with sufficient image quality for independent CoreLab analysis, e.g. allowing the acquisition of $\geq 70 \%$ of the region of interest and satisfactory blood clearing with visibility of $\geq 3$ quadrants in $\geq 70 \%$ of the pullback length.

\section{Safety parameters}

The following procedural complications (directly related to the imaging or within $24 \mathrm{~h}$ of the procedure) were prospectively collected: coronary artery dissection (angiographic) or vessel perforation, ventricular fibrillation, symptomatic brady- or tachyarrythmia, thrombus formation, spasm and air embolism.

The primary clinical safety endpoint was a composite of cardiac death, myocardial infarction and any clinically indicated revascularization. Secondary safety endpoints included death, cardiac death, myocardial infarction, Academic research consortium (ARC)-defined definite- and definite or probable stent thrombosis and major bleeding events according to the Bleeding-ARC (BARC) definition. All definitions of endpoints used in this study have been previously reported [9]. Any revascularization refers to all revascularizations [target-lesion revascularization (TLR), target-vessel revascularization (TVR), and non-TVR]. Acute renal failure was defined as an absolute increase in serum creatinine of $>1.5$ fold from baseline or a glomerular filtration rate decrease of $>25 \%$ within $72 \mathrm{~h}$.

\section{Data management}

Independent study monitors verified source data according to a prespecified monitoring plan. Data were stored in a central online database. Follow-up appointments were scheduled at 30 days, 1 and 2 years, and patients were questioned about the occurrence of angina, any adverse events. All events were independently adjudicated by a clinical event committee.

Statistical analysis

Categorical variables are presented as counts with percentages and compared using Chi square or Fisher's tests, and continuous variables as means with standard deviations or medians and interquartile ranges, and compared using the Student $t$ test and Mann-Whitney U-test, respectively. Lesion-level data were analyzed using general or generalized linear mixed models, nesting lesions within patient identifiers, wherever applicable. Time-to-first event or composite events were analyzed using Cox's regression analysis comparing the Imaging group and the control group (at 30 days, 1 and 2 years of follow-up), both crude (presented with Kaplan-Meier graphs), and adjusted (using inverse-probability of treatment weighting (IPTW), where the treatment is the imaging performed). IPTW was calculated using the following baseline variables: age, gender, body mass index, diabetes, hypertension, hypercholesterolemia, current smoker, renal insufficiency, anemia, pain onset within 6 h, Killip II, resuscitation, left ventricular 
ejection fraction, treatment of a bifurcation lesion, small vessel $\leq 2.5 \mathrm{~mm}$ diameter, long lesion $\geq 20 \mathrm{~mm}$ length, SYNTAX score, TIMI flow pre-procedure, after 20 times multiple imputation of missing data using chained equations (IPTW Adjusted Cox's Regressions weight the patients by their IPTW and are based on 20 imputed datasets). Sensitivity crude and IPTW adjusted Cox's regression analyses were conducted on the primary outcome, stratifying patients according to whether they received clopidogrel loading or prasugrel/double loading during the index procedure. Similarly, event rates at discharge were analyzed using Crude or IPTW Adjusted Poisson Regressions with robust error variances and reported as Risk Ratios RR with $95 \%$ confidence intervals (95\% CI). $p$ values from Fisher's tests are reported in case of zero events. All statistical analyses were performed with Stata 12.1 (StataCorp, Texas, USA) and differences were considered significant at $\alpha=0.05$.

\section{Results}

A total of 103 STEMI patients undergoing primary PCI were included at five European centers into the IBIS 4 imaging study between September 2009 and January 2011.

Feasibility of multimodality intracoronary imaging

The feasibility of OCT, grayscale IVUS, and IVUS-VH at baseline and follow-up imaging is shown in Table 1. Imaging of the infarct-related artery at baseline (and follow-up) was successful in $92.2 \%$ (96.6\%) patients using OCT, $93.2 \%$ (95.5\%) patients using grayscale IVUS and $88.3 \%(94.3 \%)$ patients using IVUS-VH. Imaging of the non-infarct related artery at baseline (and follow-up) was successful in $88.7 \%$ (95.6\%) patients using OCT, $90.5 \%$ $(93.3 \%)$ patients using grayscale IVUS and $83.1 \%$ $(92.6 \%)$ patients using IVUS-VH, respectively. In case an OCT pullback was successfully acquired, the frequency of OCT frames not analyzable both at baseline and follow-up was $0.3 \%$. Failure of OCT imaging was mainly related to insufficient image quality due to incomplete vessel flushing but also due to difficulties to advance the OCT catheter in the non-infarct related artery. IVUS-VH imaging failure was mainly caused by technical problems with the ECG transmission for gating of the acquisition, and by the use of manual- instead of automatic pullback.

\section{Procedural complications}

In the setting of primary PCI, in two out of 103 patients $(1.9 \%)$, the OCT pullback aquisition resulted in a complication at baseline, and in one out of 91 patients $(1.1 \%)$ at follow-up. Specifically, a dissection at the proximal stent edge was caused by the tip of the OCT imaging catheter in one of the cases at baseline; whereas the other two complications (one at baseline, one at follow-up) consisted of ventricular fibrillation induced by vessel flushing with contrast. Complications were managed by additional stent implantation in the former case, and successful defibrillation in the other cases, without further sequelae. IVUS imaging was performed without complications; and there were no cases of i.c. related thrombus formation, perforation, air embolism, or mechanical device failure or spasm (Table 2).

\section{Safety analysis}

A total of 103 imaging patients were compared to a cohort of 485 control patients.

Baseline clinical characteristics of the two groups are shown in Table 3. Imaging patients were younger, more frequently male, had a higher BMI and were less likely to have hypercholesterolemia compared to patients not undergoing i.c. imaging. Imaging patients were more often resuscitated prior to hospital admission. Prognostically important characteristics like diabetes, Killip classification and left ventricular function were similar. Procedural characteristics are shown in Table 4. Other than a lower maximal balloon pressure and a higher amount of contrast used, there was no significant difference between groups. Antithrombotic medication prior or during the procedure and at hospital discharge is shown in Table 5. Imaging patients more frequently (58.3 vs. $32.7 \%, p<0.001)$ received a concomitant loading dose of prasugrel in addition to clopidogrel and were more frequently discharged with prasugrel as compared with control patients (77.5 vs. $52.1 \%, p<0.001$ ), the proportion of patients on double antiplatelet therapy at discharge, however, was similar.

Short and long term outcomes of patients undergoing intracoronary imaging

Table 6 presents clinical outcomes at 30 days, 1 and 2 years. The primary endpoint MACE was similar for patients undergoing i.c. imaging and controls at 2 years (16.7 vs. $13.3 \%$, adjusted HR 1.4, $95 \%$ CI 0.77-2.52, $p=0.27$ ) (Fig. 1). Similarly, no differences in the individual endpoints including death, myocardial infarction and any revascularization were observed at 2 years (Fig. 2). Two recurrent myocardial infarctions were noted in the imaging group within 30 days. One event was related to an early stent thrombosis 4 days after stent implantation in a calcified ostial RCA lesion due to stent underexpansion. The second event occurred in a patient who was readmitted with cardiogenic shock due to ischemic mitral regurgitation 
Table 1 Feasibility of OCT, IVUS, and IVUS-VH at baseline and 13 months follow-up. Bold numbers indicate the overall sucess of i.c. imaging

\begin{tabular}{|c|c|c|c|c|c|c|}
\hline & \multicolumn{3}{|c|}{ Infarct-related vessel } & \multicolumn{3}{|c|}{ Non-infarct-related vessel } \\
\hline & OCT & IVUS & IVUS-VH & OCT & IVUS & IVUS-VH \\
\hline \multicolumn{7}{|l|}{ Baseline } \\
\hline Number of patients & 103 & & & 103 & & \\
\hline Number of vessels available for imaging & 103 & 103 & 103 & 204 & 201 & 201 \\
\hline Successful imaging & $95(92.2)$ & $96(93.2)$ & $91(88.3)$ & $181(88.7)$ & $181(90.5)$ & $172(83.1)$ \\
\hline Unsuccessful imaging & $8(7.8)$ & $7(6.8)$ & $12(11.7)$ & $23(11.3)$ & $20(9.5)$ & $29(14.4)$ \\
\hline Failure to cross lesion & $1(1.0)$ & $3(2.9)$ & $3(2.9)$ & $11(5.4)$ & $9(4.5)$ & $9(4.5)$ \\
\hline Technical problem ${ }^{\mathrm{c}}$ & 0 & $1(1.0)$ & $6(5.8)$ & 0 & $3(1.5)$ & $12(6.0)$ \\
\hline Insufficient quality for analysis & $6(5.8)$ & $2(1.9)$ & $2(1.9)$ & $10(4.9)$ & $6(3.0)$ & $6(3.0)$ \\
\hline Patient compliance $^{\mathrm{d}}$ & $1(1.0)$ & $1(1.0)$ & $1(1.0)$ & $2(1.0)$ & $2(1.0)$ & $2(1.0)$ \\
\hline \multicolumn{7}{|l|}{ Follow-up } \\
\hline Number of patients & 91 & & & 91 & & \\
\hline Total number of vessels available for imaging & 91 & 91 & 91 & 185 & 183 & 183 \\
\hline Not obtained as at baseline ${ }^{a}$ & 2 & 2 & 2 & 25 & 20 & 20 \\
\hline Successful imaging $^{\mathrm{b}}$ & $86(96.6)$ & $85(95.5)$ & $84(94.3)$ & $153(95.6)$ & $152(93.3)$ & $150(92.6)$ \\
\hline Unsuccessful imaging & $3(3.4)$ & $4(3.4)$ & $5(5.6)$ & $7(4.4)$ & $9(5.5)$ & $11(7.3)$ \\
\hline Failure to cross lesion & $2(2.2)$ & $2(2.2)$ & $2(2.2)$ & 0 & $1(0.6)$ & $1(0.6)$ \\
\hline Technical problem ${ }^{\mathrm{c}}$ & 0 & $2(1.1)$ & $3(3.4)$ & $1(0.6)$ & $5(3.1)$ & $7(4.3)$ \\
\hline Insufficient quality for analysis & $1(1.1)$ & 0 & 0 & $3(1.9)$ & $1(0.6)$ & $1(0.6)$ \\
\hline Patient compliance $^{\mathrm{d}}$ & 0 & 0 & 0 & $3(1.9)$ & $2(1.2)$ & $2(1.2)$ \\
\hline
\end{tabular}

Values are number $(\%)$ or mean $\pm \mathrm{SD}$

OCT optical coherence tomography, IVUS intravascular ultrasound, $V H$ virtual histology

${ }^{a}$ Not investigated at follow-up as no baseline was available

b The success rate at follow-up = successful imaging/(total number of vessel available - number of not obtained as at baseline)

c Technical problem is defined as manual pullback or DVD defect or ECG trigger missing

${ }^{\mathrm{d}}$ Patient compliance is defined as preterm termination of the imaging procedure due to patient discomfort in view of the prolonged catheterization time

Table 2 Complications related to imaging procedure (per patient)

\begin{tabular}{|c|c|c|c|c|}
\hline & \multicolumn{2}{|l|}{ Baseline } & \multicolumn{2}{|l|}{ Follow-up } \\
\hline & OCT $\mathrm{N}=103$ & IVUS $\mathrm{N}=103$ & OCT $\mathrm{N}=91$ & IVUS $\mathrm{N}=91$ \\
\hline Dissection & $1(1.0)$ & 0 & 0 & 0 \\
\hline Ventricular fibrillation & $1(1.0)$ & 0 & $1(1.1)$ & 0 \\
\hline Symptomatic brady- or tachyarrhythmia & 0 & 0 & 0 & 0 \\
\hline Thrombus formation & 0 & 0 & 0 & 0 \\
\hline Perforation & 0 & 0 & 0 & 0 \\
\hline Air embolism & 0 & 0 & 0 & 0 \\
\hline Total & $2(1.9)$ & 0 & $1(1.1)$ & 0 \\
\hline
\end{tabular}

Values are number $(\%)$

OCT optical coherence tomography, IVUS intravascular ultrasonography

with documentation of stent thrombosis. There was no significant difference in the rate of acute renal failure at 30 days between 2 groups ( 1 vs. $1.9 \%$, adjusted HR 0.68,
$95 \%$ CI $0.09-5.37, p=0.72$ ). Revascularization of noninfarct related arteries (clinically indicated non-TVR) is an important surrogate for lesion progression potentially 
Table 3 Baseline clinical characteristics

\begin{tabular}{|c|c|c|c|}
\hline & Imaging group $(\mathrm{n}=103)$ & Control group $(\mathrm{n}=485)$ & $p$ value \\
\hline Age (yrs) & $58.2 \pm 10.5$ & $61.5 \pm 12.2$ & 0.012 \\
\hline Male (gender) & $93(90.3)$ & $385(79.4)$ & 0.008 \\
\hline BMI $\left(\mathrm{kg} / \mathrm{m}^{2}\right)$ & $27.8 \pm 4.2$ & $26.8 \pm 4.0$ & 0.019 \\
\hline \multicolumn{4}{|l|}{ Cardiovascular risk factors } \\
\hline Diabetes & $13(12.6)$ & $65(13.4)$ & 1.00 \\
\hline Hypertension & $48(46.6)$ & $212(43.7)$ & 0.66 \\
\hline Hypercholesterolemia & $42(40.8)$ & $259(53.7)$ & 0.022 \\
\hline Current smoker & $48(46.6)$ & $242(50.4)$ & 0.52 \\
\hline Family history of CAD & $31(31.0)$ & $130(27.3)$ & 0.46 \\
\hline Renal failure & $1(1.0)$ & $13(2.7)$ & 0.48 \\
\hline Previous MI & $2(1.9)$ & $26(5.4)$ & 0.20 \\
\hline Previous CABG & $0(0.0)$ & $13(2.7)$ & 0.14 \\
\hline Peak CK & $1847(1045-3494)$ & $1652(800-2814)$ & 0.20 \\
\hline Peak CK-MB & $187(107-319)$ & $160(72-300)$ & 0.20 \\
\hline \multicolumn{4}{|l|}{ Clinical presentation } \\
\hline Time from symptom onset to balloon inflation (min) & $258(170-472)$ & $244(164-422)$ & 0.72 \\
\hline Time from arrival at hospital to balloon inflation (min) & $40(30-56)$ & $42(30-63)$ & 0.18 \\
\hline Killip class II, III, or IV & $8(7.8)$ & $34(7.0)$ & 0.83 \\
\hline Resuscitation before hospital arrival & $5(4.9)$ & $7(1.4)$ & 0.042 \\
\hline LVEF $(\%)$ & $47.8 \pm 9.4$ & $48.6 \pm 10.1$ & 0.52 \\
\hline
\end{tabular}

Values are expressed as mean \pm SD or number $(\%)$

$B M I$ body mass index, $C A D$ coronary artery disease, $M I$ myocardial infarction, $P C I$ percutaneous coronary intervention, $C A B G$ coronary artery bypass graft, $C K$ creatine kinase, $L V E F$ left ventricular ejection fraction

related to i.c. imaging. Non-TVR occurred at a similar frequency in the imaging $(9.0 \%)$ and control group (4.8 \%, HR 1.70, $95 \%$ CI 0.76-3.81, $p=0.20)$. Similarly, there was no difference in the frequency of any clinically indicated revascularization (Table 6).

To investigate whether the observed differences in the use of P2Y12 inhibitors were responsible similar MACE rates, we performed a sensitivity analysis for the primary endpoint MACE. We found hazards to be consistent for both groups at any time point of follow-up when separately comparing patients receiving a clopidogrel loading dose (imaging vs. control group, adjusted 2 year HR 1.18, $95 \%$ CI $0.39-3.61, p=0.77$ ) and those receiving either prasugrel or a double loading dose separately (adjusted 2 year HR $1.6795 \%$ CI 0.81-3.43, $p=0.16$ ) (Appendix Table S1).

\section{Discussion}

This is the first report on the feasibility and safety of multimodality three-vessel intracoronary imaging in the setting of a prospective observational study in STEMI patients undergoing primary PCI. Less extensive intracoronary imaging may be used in daily clinical routine, a situation for which our findings are also applicable.
The following major findings apply to this study:

1. Multimodality three-vessel i.c. imaging was consistent with high degree of success, confirming the feasibility even in the acute setting of primary PCI at centers with various level of expertise in i.c. imaging.

2. Periprocedural complications were rare $(1.9 \%$ per patient at baseline, $1.1 \%$ at follow-up), solely related to the imaging with OCT, and resolved without sequelae in all cases.

3. Rates of death and myocardial infarction at 30 days were similar between patients undergoing i.c. imaging and controls attesting to the procedural safety of threevessel multimodality imaging.

4. Rates of MACE up to 2 years were low and similar for both groups indicating that diagnostic serial multimodality i.c. imaging is not associated with harm.

Feasibility

The present study demonstrates an overall high feasibility of both OCT and IVUS for imaging the infarct vessel, which did not differ between the baseline acute setting and the planned follow-up investigation at 13 months, suggesting that the acute presentation does not limit the success rate of i.c. imaging except in cases of hemodynamic 
Table 4 Baseline procedural characteristics

\begin{tabular}{|c|c|c|c|}
\hline & Imaging group $(\mathrm{n}=103)$ & Control group $(\mathrm{n}=485)$ & $p$ value \\
\hline No of lesions treated in infarct vessel & 121 & 542 & \\
\hline Infarct vessel location & & & $0.24 *$ \\
\hline Left main coronary artery & $1(1.0)$ & $1(0.2)$ & \\
\hline Left anterior descending artery & $46(44.7)$ & $184(38.0)$ & \\
\hline Left circumflex artery & $18(17.5)$ & $68(14.0)$ & \\
\hline Right coronary artery & 38 (36.9) & $229(47.3)$ & \\
\hline Baseline TIMI flow & & & $0.51 *$ \\
\hline 0 or 1 & $73(60.8)$ & $351(65.0)$ & \\
\hline 2 & $17(14.2)$ & $67(12.4)$ & \\
\hline 3 & $30(25.0)$ & $122(22.6)$ & \\
\hline \multicolumn{4}{|l|}{ Lesion complexity } \\
\hline Bifurcation lesion,no. & $14(13.6)$ & $44(9.1)$ & $0.20 *$ \\
\hline Small vessel & $14(13.6)$ & $67(13.9)$ & $1.00 *$ \\
\hline Long lesion & $31(30.1)$ & $149(31.0)$ & $0.91 *$ \\
\hline SYNTAX MI Score & $14.7 \pm 6.7$ & $14.5 \pm 8.3$ & $0.82 *$ \\
\hline \multicolumn{4}{|l|}{ Primary PCI procedure } \\
\hline No. of stents per lesion & $1.22 \pm 0.51$ & $1.30 \pm 0.67$ & $0.28 *$ \\
\hline \multicolumn{4}{|l|}{ Type of stent } \\
\hline Biolimus-eluting stent & $64(52.9)$ & $268(49.8)$ & $0.60 *$ \\
\hline Bare metal stent & $57(47.1)$ & $274(50.9)$ & $0.57 *$ \\
\hline Stent length per lesion (mm) & $22.1 \pm 10.5$ & $24.1 \pm 13.2$ & $0.16^{*}$ \\
\hline Stent diameter $(\mathrm{mm})$ & $3.25 \pm 0.49$ & $3.2 \pm 0.7$ & $0.36^{*}$ \\
\hline Direct stenting & $24(19.8)$ & $127(23.6)$ & $0.31 *$ \\
\hline Maximal balloon pressure (atm) & $13.7 \pm 2.9$ & $14.9 \pm 3.4$ & $<0.001^{*}$ \\
\hline Thrombus aspiration performed & $70(68.0)$ & $288(59.5)$ & 0.12 \\
\hline Intravenous vasopressors & $2(1.9)$ & $14(2.9)$ & 1.00 \\
\hline TIMI flow post-procedure & & & 0.76 \\
\hline 0 or 1 & $0(0.0)$ & $3(0.6)$ & \\
\hline 2 & $2(1.7)$ & $22(4.1)$ & \\
\hline 3 & $119(98.3)$ & $516(95.4)$ & \\
\hline \multicolumn{4}{|c|}{ Total amount of contrast used during primary PCI (ml) } \\
\hline BL & $268.3 \pm 109.8$ & $197.9 \pm 88.8$ & $<0.001$ \\
\hline \multicolumn{4}{|l|}{ Total amount of contrast used for imaging (ml) } \\
\hline BL & $174 \pm 72$ & n.a. & \\
\hline FUP & $169 \pm 83$ & n.a. & \\
\hline Total time for primary PCI intervention (min) & $34.3 \pm 18.5$ & $36.2 \pm 20.1$ & 0.39 \\
\hline Total time used for imaging (min) & $44 \pm 22$ & n.a. & \\
\hline
\end{tabular}

Data are expressed as number $(\%)$ unless otherwise specified

$I Q R$ interquartile range, $S D$ standard deviation, $P C I$ percutaneous coronary intervention, $Q C A$ quantitative coronary angiography, TIMI thrombolysis in myocardial infarction, $B L$ baseline, FUP follow-up, $M I$ myocardial infarction, n.a. not applicable

* Mixed model $p$ value accounting for lesions nested within patient

instability or poor PCI result. As opposed to the infarctrelated vessels, the success rates for evaluating non-infarct related arteries were lower. The principal reason for this was the caliber of some of the arteries, vessel tortuosity, and an untreated stenosis. This is however, not surprising since these features typically make part of the exclusion criteria in OCT studies. Nevertheless, as we aimed at a three-vessel evaluation in an already diseased patient population, with as few exclusion criteria as possible to minimize selection bias, these vessels were included yet still contribute to an overall high success rate. Studies investigating the feasibility of multimodality three-vessel i.c. imaging in STEMI patients undergoing primary PCI are lacking. Up until now, few studies performed three vessel 
Table 5 Medication during the procedure and at discharge

\begin{tabular}{|c|c|c|c|}
\hline & Imaging group $(\mathrm{n}=103)$ & Control group $(\mathrm{n}=485)$ & $p$ value \\
\hline \multicolumn{4}{|l|}{ Periprocedural medication } \\
\hline Unfractionated heparin & $101(98.1)$ & $478(98.6)$ & 0.66 \\
\hline Bivalirudin & $6(5.8)$ & $29(6.0)$ & 1.00 \\
\hline Glycoprotein IIb/IIIa antagonists & $54(52.4)$ & $241(49.7)$ & 0.67 \\
\hline \multicolumn{4}{|l|}{ Loading dose of clopidogrel and prasugrel } \\
\hline Clopidogrel only (600 mg) & $26(25.2)$ & $251(52.0)$ & $<0.001$ \\
\hline Prasugrel only (60 mg) & $17(16.5)$ & $68(14.1)$ & 0.54 \\
\hline Both & $60(58.3)$ & $158(32.7)$ & $<0.001$ \\
\hline No loading dose clopidogrel and prasugrel & $0(0.0)$ & $6(1.2)$ & 0.60 \\
\hline \multicolumn{4}{|l|}{ Medication at discharge } \\
\hline Aspirin & $102(100.0)$ & $481(99.8)$ & 1.00 \\
\hline Clopidogrel & $23(22.5)$ & $230(47.7)$ & $<0.001$ \\
\hline Prasugrel & $79(77.5)$ & $251(52.1)$ & $<0.001$ \\
\hline Any dual antiplatelet therapy & $102(100.0)$ & $480(99.6)$ & 1.00 \\
\hline
\end{tabular}

Values are $\mathrm{n}(\%)$

(single modality) imaging with either OCT [10] or IVUS [11], however, these reports did not disclose the number of excluded patients related to imaging failures, thus only reported the number of pullbacks excluded due to insufficient imaging quality and safety data is not available.

\section{Periprocedural complications}

Peri-procedural complications were rare and solely related to the use of OCT. In two out of 103 OCT pullbacks (1.9\%), ventricular fibrillation occurred due to prolonged flushing with contrast media. Given the overall low frequency of ventricular fibrillation in this patient group presenting for primary PCI, and in view of the prompt restoration of sinus rhythm following defibrillation, OCT can be considered as a safe diagnostic tool. Nonetheless, physicians using this technology need to be aware about the potential of inducing ventricular fibrillation. The single dissection recorded at a proximal stent edge was related to the short monorail of the previous version of the dragonfly OCT catheter, which was difficult to advance in a vessel curvature.

The largest study with time-domain (TD-) OCT where peri-procedural complications were reported included 468 mainly stable coronary artery disease patients, and observed ventricular fibrillation in $1.1 \%$ of patients, air embolism in $0.6 \%$ and vessel dissection in $0.2 \%$ of patients. Only few data is available on the safety of FD-OCT $[12,13]$, which acquires images at frame rates at least 10 times faster than TD-OCT and results in an improved imaging quality. The marked simplification of the acquisition procedures and reduction in the required contrast volume has decreased the imaging procedure time and increased the imaging quality [11]. The absence of complications with the use of IVUS confirms the already known high degree of safety of this technique, and may be the result of device adaptations since the introduction of the technique in the early 1990s and the high degree of experience with its use. Conversely, OCT has been introduced only 15 years later. In our study, two participating study centers initiated the use of OCT only with the occasion of this study, which increases the applicability of the study results to a broader spectrum of centers performing i.c. imaging.

\section{Short and long-term safety}

We did not limit our analysis to peri-procedural complications and compared clinical outcome measures of safety between imaging patients and a control group. At 30 days, the composite endpoint cardiac death or MI occurred at a similar rate in imaging and non-imaging patients, further supporting the procedural and long-term safety of multimodality imaging for documentary purpose. Similarly, no difference in cardiac death or MI was noted at 1 and 2 years of follow-up.

Safety concerns related to imaging procedures in native coronary arteries are not limited to peri-procedural complications occurring during the first 30 days after imaging but also apply to a hypothetical acceleration of 
Table 6 Clinical outcomes at discharge, 30 days, 1, and 2 years

\begin{tabular}{|c|c|c|c|c|c|c|}
\hline & \multirow{2}{*}{$\begin{array}{l}\text { Imaging group } \\
(\mathrm{n}=103)\end{array}$} & \multirow{2}{*}{$\begin{array}{l}\text { Control group } \\
(\mathrm{n}=485)\end{array}$} & \multicolumn{2}{|l|}{ Crude } & \multicolumn{2}{|l|}{ IPTW adjusted } \\
\hline & & & $\begin{array}{l}\text { Hazard ratio } \\
(95 \% \mathrm{CI})\end{array}$ & $p$ value & $\begin{array}{l}\text { Hazard ratio } \\
(95 \% \mathrm{CI})\end{array}$ & $p$ value \\
\hline \multicolumn{7}{|l|}{ Clinical events at 30 days $^{\mathrm{a}}$} \\
\hline Death & $1(1.0)$ & $8(1.7)$ & $0.59(0.07-4.69)$ & 0.62 & $1.20(0.15-9.47)$ & 0.86 \\
\hline Cardiac death & $1(1.0)$ & $8(1.7)$ & $0.59(0.07-4.69)$ & 0.62 & $1.20(0.15-9.47)$ & 0.86 \\
\hline MI & $2(2.0)$ & $5(1.0)$ & $1.89(0.37-9.73)$ & 0.45 & $2.66(0.51-13.85)$ & 0.25 \\
\hline Death or MI & $3(2.9)$ & $13(2.7)$ & $1.09(0.31-3.83)$ & 0.89 & $1.79(0.50-6.34)$ & 0.37 \\
\hline Cardiac death or MI & $3(2.9)$ & $13(2.7)$ & $1.09(0.31-3.83)$ & 0.89 & $1.79(0.50-6.34)$ & 0.37 \\
\hline $\begin{array}{l}\text { Clinically indicated } \\
\text { revascularization (any) }\end{array}$ & $2(2.0)$ & $7(1.5)$ & $1.35(0.28-6.50)$ & 0.71 & $1.82(0.37-8.85)$ & 0.46 \\
\hline Clinically indicated non-TVR & $0(0.0)$ & $1(0.2)$ & $2.34(0.21-25.57)$ & 1.00 & . & . \\
\hline Clinically indicated TVR & $2(2.0)$ & $7(1.5)$ & $1.35(0.28-6.50)$ & 0.71 & $1.82(0.37-8.85)$ & 0.46 \\
\hline Clinically indicated TLR & $2(2.0)$ & $7(1.5)$ & $1.35(0.28-6.50)$ & 0.71 & $1.82(0.37-8.85)$ & 0.46 \\
\hline MACE $^{\mathrm{a}}$ & $3(2.9)$ & $16(3.3)$ & $0.88(0.26-3.03)$ & 0.84 & $1.43(0.41-4.98)$ & 0.57 \\
\hline Definite ST & $2(2.0)$ & $6(1.2)$ & $1.58(0.32-7.82)$ & 0.58 & $2.10(0.42-10.50)$ & 0.37 \\
\hline Definite or probable ST & $2(2.0)$ & $11(2.3)$ & $0.86(0.19-3.88)$ & 0.84 & $1.22(0.27-5.61)$ & 0.79 \\
\hline Any major bleeding (BARC $3,4,5$ ) & $4(3.9)$ & $15(3.1)$ & $1.25(0.42-3.78)$ & 0.69 & $2.14(0.57-7.69)$ & 0.26 \\
\hline Acute renal failure & $1(1.0)$ & $9(1.9)$ & $0.52(0.07-4.12)$ & 0.54 & $0.68(0.09-5.37)$ & 0.72 \\
\hline \multicolumn{7}{|l|}{ Clinical events at 1 year $^{\mathrm{a}}$} \\
\hline Death & $2(2.0)$ & $12(2.5)$ & $0.78(0.17-3.48)$ & 0.74 & $1.08(0.21-5.45)$ & 0.92 \\
\hline Cardiac death & $2(2.0)$ & $10(2.1)$ & $0.93(0.20-4.27)$ & 0.93 & $1.29(0.25-6.60)$ & 0.76 \\
\hline MI & $5(4.9)$ & $13(2.8)$ & $1.81(0.65-5.08)$ & 0.26 & $1.74(0.57-5.29)$ & 0.33 \\
\hline Death or MI & $6(5.9)$ & $24(5.0)$ & $1.18(0.48-2.88)$ & 0.72 & $1.34(0.50-3.56)$ & 0.56 \\
\hline Cardiac death or MI & $6(5.9)$ & $22(4.6)$ & $1.29(0.52-3.17)$ & 0.58 & $1.45(0.54-3.89)$ & 0.46 \\
\hline $\begin{array}{l}\text { Clinically indicated } \\
\text { revascularization (any) }\end{array}$ & $6(5.9)$ & $29(6.2)$ & $0.96(0.40-2.32)$ & 0.93 & $0.89(0.34-2.33)$ & 0.82 \\
\hline Clincally indicated non-TVR & $4(4.0)$ & $11(2.4)$ & $1.70(0.54-5.34)$ & 0.36 & $1.29(0.39-4.25)$ & 0.68 \\
\hline Clinically indicated TVR & $3(3.0)$ & $22(4.7)$ & $0.63(0.19-2.11)$ & 0.45 & $0.77(0.22-2.75)$ & 0.69 \\
\hline Clinically indicated TLR & $3(3.0)$ & $22(4.7)$ & $0.63(0.19-2.11)$ & 0.45 & $0.77(0.22-2.75)$ & 0.69 \\
\hline $\mathrm{MACE}^{\mathrm{b}}$ & $7(6.8)$ & $39(8.2)$ & $0.83(0.37-1.87)$ & 0.66 & $0.90(0.37-2.20)$ & 0.83 \\
\hline Definite ST & $3(3.0)$ & $8(1.7)$ & $1.77(0.47-6.66)$ & 0.40 & $2.01(0.51-7.92)$ & 0.32 \\
\hline Definite or probable ST & $3(3.0)$ & $15(3.1)$ & $0.94(0.27-3.25)$ & 0.92 & $1.15(0.31-4.17)$ & 0.84 \\
\hline Any major bleeding (BARC 3,4,5) & $5(4.9)$ & $23(4.8)$ & $1.02(0.39-2.68)$ & 0.97 & $1.60(0.50-5.10)$ & 0.43 \\
\hline \multicolumn{7}{|l|}{ Clinical events at 2 years $^{\mathrm{a}}$} \\
\hline Death & $2(2.0)$ & $19(4.0)$ & $0.49(0.11-2.09)$ & 0.33 & $0.68(0.14-3.35)$ & 0.64 \\
\hline Cardiac death & $2(2.0)$ & $13(2.7)$ & $0.72(0.16-3.17)$ & 0.66 & $1.00(0.20-5.02)$ & 1.00 \\
\hline MI & $5(4.9)$ & $21(4.5)$ & $1.11(0.42-2.95)$ & 0.83 & $1.07(0.37-3.11)$ & 0.90 \\
\hline Death or MI & $6(5.9)$ & $39(8.2)$ & $0.72(0.30-1.70)$ & 0.45 & $0.82(0.32-2.14)$ & 0.69 \\
\hline Cardiac death or MI & $6(5.9)$ & $33(7.0)$ & $0.85(0.36-2.03)$ & 0.72 & $0.97(0.37-2.53)$ & 0.95 \\
\hline $\begin{array}{l}\text { Clinically indicated } \\
\text { revascularization (any) }\end{array}$ & $16(15.9)$ & $49(10.6)$ & $1.54(0.87-2.70)$ & 0.14 & $1.60(0.86-2.96)$ & 0.14 \\
\hline Clinically indicated non-TVR & $9(9.0)$ & $22(4.8)$ & $1.93(0.89-4.20)$ & 0.096 & $1.70(0.76-3.81)$ & 0.20 \\
\hline Clinically indicated TVR & $10(10.0)$ & $36(7.8)$ & $1.28(0.64-2.58)$ & 0.49 & $1.52(0.71-3.26)$ & 0.28 \\
\hline Clinically indicated TLR & $9(9.0)$ & $34(7.3)$ & $1.22(0.59-2.54)$ & 0.60 & $1.46(0.65-3.26)$ & 0.36 \\
\hline $\mathrm{MACE}^{\mathrm{a}}$ & $17(16.7)$ & $63(13.3)$ & $1.27(0.74-2.16)$ & 0.39 & $1.40(0.77-2.52)$ & 0.27 \\
\hline Definite ST & $3(3.0)$ & $11(2.3)$ & $1.28(0.36-4.58)$ & 0.71 & $1.46(0.38-5.51)$ & 0.58 \\
\hline Definite or probable ST & $3(3.0)$ & $20(4.2)$ & $0.70(0.21-2.36)$ & 0.56 & $0.85(0.24-3.02)$ & 0.80 \\
\hline
\end{tabular}


Table 6 continued

\begin{tabular}{|c|c|c|c|c|c|c|}
\hline & \multirow{2}{*}{$\begin{array}{l}\text { Imaging group } \\
(\mathrm{n}=103)\end{array}$} & \multirow{2}{*}{$\begin{array}{l}\text { Control group } \\
(\mathrm{n}=485)\end{array}$} & \multicolumn{2}{|l|}{ Crude } & \multicolumn{2}{|l|}{ IPTW adjusted } \\
\hline & & & $\begin{array}{l}\text { Hazard ratio } \\
(95 \% \mathrm{CI})\end{array}$ & $p$ value & $\begin{array}{l}\text { Hazard ratio } \\
(95 \% \mathrm{CI})\end{array}$ & $p$ value \\
\hline Any major bleeding (BARC $3,4,5$ ) & $5(4.9)$ & $24(5.0)$ & $0.97(0.37-2.55)$ & 0.96 & $1.52(0.48-4.83)$ & 0.48 \\
\hline
\end{tabular}

Data are number of first events (\%) and rate ratios RR (95\% CI) with $p$ values from Poisson Regressions Crude, or IPTW Adjusted, using robust error variances

CI confidence interval

a Data are number of first events (\% from Kaplan-Meier life-tables) and hazard ratios HR (95\% CI) with $p$-values from Cox's Regressions Crude, or IPTW Adjusted

b MACE composite of all cardiac death, myocardial infarciton and any clinically indicated revascularization

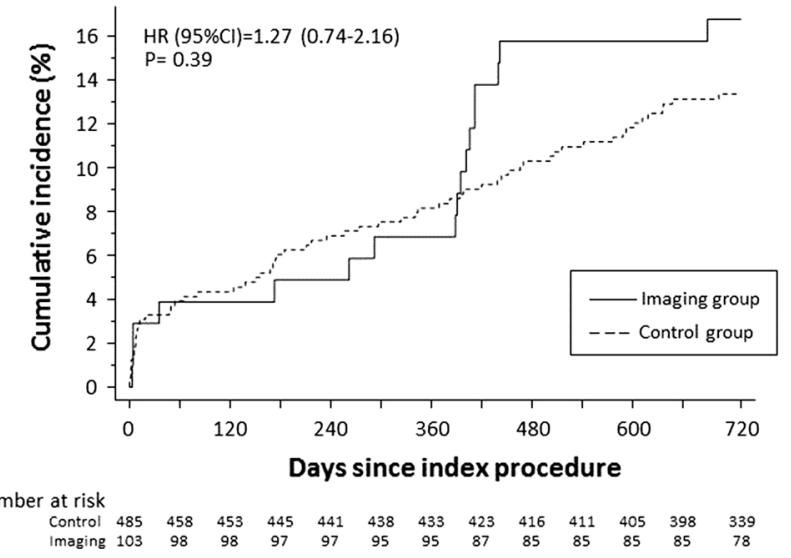

Fig. 1 Cumulative incidence curve for MACE (cardiac death, MI and any clinically indicated revascularization) throughout 2 years. Continuous line indicates imaging group, dotted line indicates control group not undergoing imaging

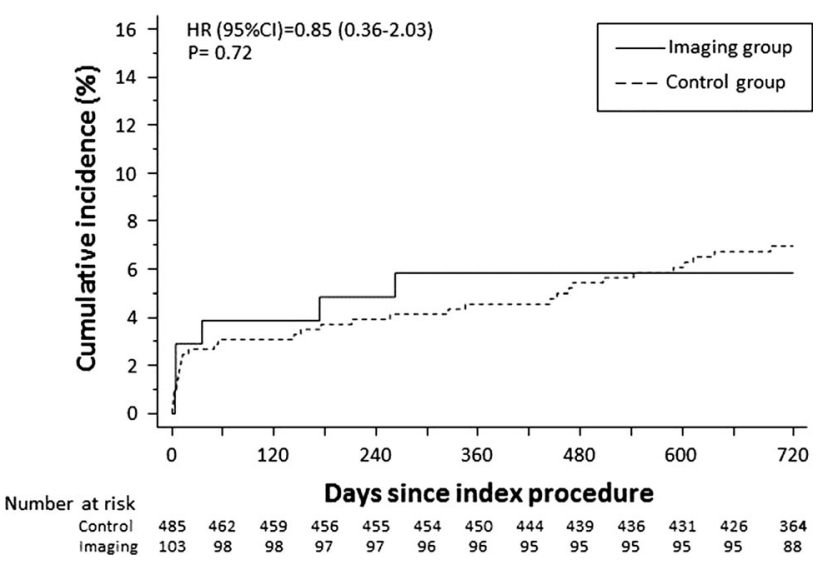

Fig. 2 Cumulative incidence curve for the composite of cardiac death or MI throughout 2 years

atherosclerosis during long-term follow-up [14-16] Hypothetically, the introduction of imaging catheters solely for diagnostic purposes (IVUS: 2.9 French, OCT: 2.7 French) may induce clinically silent endothelial injury as previously demonstrated in an experimental model [17].
The iatrogenic damage may trigger atherosclerotic disease progression by initiating clinically silent plaque ruptures with subsequent progression [18]. As non-infarct related arteries of STEMI patients are known to accommodate vulnerable plaques, and as they are potentially more sensitive to catheter-induced rupture than other lesions, we extended our evaluation to a long-term monitoring with a particular focus on repeat revascularization events in untreated segments [10, 11]., Indicators of a disease progression in untreated vessel segments are the frequency of target vessel revascularizations (TVR) and the revascularizations occurring in the untreated vessels (non-target lesion revascularization, non-TVR). Throughout 2 years, TVR and non-TVR occurred in a similar frequency when comparing the imaging with the control group. As shown in Fig. 2, the cumulative incidence curve for MACE diverges at the timepoint of the angiographic follow-up, which is solely driven by a non-significant increase in repeat revascularization procedures not directly associated with intracoronary imaging but rather related to the welldocumented occulo-stenotic reflex [19]. Similar to our findings, the "Avasimibe and progression of coronary lesions assessed by IVUS" (A-PLUS trial) investigating i.c. imaging related atherosclerosis progression, found no evidence by quantitative coronary analysis of disease progression 24 months following IVUS investigation, as compared to control arteries of the same patients [15].

OCT and IVUS require the placement of an i.c. guidewire and therefore, radiation exposure and contrast agents are important safety considerations. While we did not record the incremental radiation dosage required for threevessel i.c. imaging, we did assess the amount of contrast agents used. OCT requires complete blood removal achieved by a proper contrast injection. In our study, the contrast use per pullback ranged between 15 and $30 \mathrm{ml}$ per pullback and we used injection rates between 3.5 and $6 \mathrm{ml} /$ sec. A total of $174 \mathrm{ml}$ of contrast media was injected at baseline and $164 \mathrm{ml}$ at follow-up. This relatively high number has to be interpreted in light of our IBIS-4 study 
protocol, which required the placement of a guidewire in two non-intervened vessels, the placement and documentation of the image wire for matching purpose, the acquisition of OCT pullbacks in three vessels and a documentary final angiography after both IVUS and OCT to confirm the absence of harm. Despite the higher contrast use in imaging patients, the risk of developing renal failure after primary PCI was low and similar for patients in the imaging and control group (1.0 vs. $1.9 \%, p=0.72$ ). In addition, no difference was observed in the creatinine increase from admission to peak during the index hospitalization $(7.2 \pm 9.2$ vs. $8.8 \pm 15.2 \mu \mathrm{mol} / \mathrm{L}, p=0.16)$.

\section{Limitation}

This study has some limitations. First of all, there is to date no clinical indication for the performance of multi-modality three vessel imaging during primary PCI. However, the results observed in the setting of a maximal i.c. imaging approach can be translated to less extensive imaging procedures in daily clinical routine, attesting to their feasibility and safety.

Despite the attempt to select a comparable control group, the decision to perform i.c. imaging was not random introducing some selection bias and probably including a lower risk population in the imaging study. Repeated manipulation with the infarct related artery could potentially lead to an increasing risk for micro-embolization. Whilst myocardial blush grade was not recorded, no difference in final TIMI flow between the two groups was recorded. Although the presence of a myocardial infarction at baseline hypothetically masks the occurrence of an imaging induced peri-procedural myocardial infarction, we did not observe an increased risk at follow-up under stable conditions. In addition, the follow-up angiography and imaging procedures might increase the event rates in imaging group, however, no difference in clinical outcomes at 2 years has been observed between two groups.

In addition, we did not assess the radiation dose in both groups. Nevertheless, the total radiation time inevitably increases as positioning of the coronary wire and imaging catheter has to be documented with both OCT and IVUS. The total number of patients investigated is limited considering the presence of a complex imaging protocol.

\section{Conclusions}

Multi-modality three-vessel i.c. imaging in the acute setting of STEMI and performed for documentary purposes is consistent with a high degree of success and can be performed safely without impact on cardiovascular events at long-term follow-up. This data provides reassurance on the feasibility and safety of intravascular imaging in daily routine, where a less extensive approach may be used.

Conflict of interest The authors report the following conflicts of interest/financial disclosures: Dr. Räber has received speaker fees and research support from St. Jude Medical. Prof. Meier has received has received educational and research support to the institution from Abbott, Cordis, Boston Scientific, and Medtronic. Prof. Windecker has received research contracts to the institution from Biotronic and St. Jude. Prof. Wenaweser has received honoraria and lecture fees from Medtronic and Edwards Lifesciences. Prof. Roffi reported receiving grants from Boston Scientific, Abbott Vascular, Medtronic, and Biosensor; and payment for lectures from Lilly-Daiichi Sankyo. Prof. Lüscher reports research grants to the institution from Biosensors, Biotronik, Boston Scientific, and Medtronic All other authors reported no conflicts of interest.

\section{References}

1. Tearney GJ, Regar E, Akasaka T et al (2012) Consensus standards for acquisition, measurement, and reporting of intravascular optical coherence tomography studies: a report from the international working group for intravascular optical coherence tomography standardization and validation. J Am Coll Cardiol 59:1058-1072

2. Mintz GS, Nissen SE, Anderson WD et al (2001) American College of Cardiology Clinical Expert Consensus Document on Standards for Acquisition, Measurement and Reporting of Intravascular Ultrasound Studies (IVUS). A report of the American College of Cardiology Task Force on Clinical Expert Consensus Documents. J Am Coll Cardiol 37:1478-1492

3. Nair A, Kuban BD, Tuzcu EM et al (2002) Coronary plaque classification with intravascular ultrasound radiofrequency data analysis. Circulation 106:2200-2206

4. Sawada T, Shite J, Garcia-Garcia HM et al (2008) Feasibility of combined use of intravascular ultrasound radiofrequency data analysis and optical coherence tomography for detecting thin-cap fibroatheroma. Eur Heart J 29:1136-1146

5. Trii S NG, Ijichi T, Yoshikawa A, Ikari Y (2013) Ex vivo assessment of plaque characteristics with optical frequency domain imaging; accuracy and pitfalls in diagnosis of lipid rich plaque. Abstract Presented at ESC

6. Witzenbichler B, Maehara A, Weisz G et al (2014) Relationship between intravascular ultrasound guidance and clinical outcomes after drug-eluting stents: the assessment of dual antiplatelet therapy with drug-eluting stents (ADAPT-DES) study. Circulation 129:463-470

7. Räber L, Zaugg S, Kelbaek H, Roffi M, Holmvang L (2014) Effect of high-intensity statin therapy no atherosclerosis in noninfarct related coronary arteries (IBIS-4): a serial intravascular ultrasonography study. Eur Heart J. doi:10.1093/eurheartj/ehu373

8. Räber L, Kelbaek H, Ostojic M et al (2012) Effect of biolimuseluting stents with biodegradable polymer vs bare-metal stents on cardiovascular events among patients with acute myocardial infarction: the COMFORTABLE AMI randomized trial. J Am Med Assoc 308:777-787

9. Raber L, Kelbaek H, Ostoijc M et al (2012) Comparison of biolimus eluted from an erodible stent coating with bare metal stents in acute ST-elevation myocardial infarction (COMFORTABLE AMI trial): rationale and design. EuroIntervention $\mathrm{J}$ EuroPCR Collab Work Group Interv Cardiol Eur Soc Cardiol $7: 1435-1443$ 
10. Kato K, Yonetsu T, Kim SJ et al (2012) Nonculprit plaques in patients with acute coronary syndromes have more vulnerable features compared with those with non-acute coronary syndromes: a 3-vessel optical coherence tomography study. Circ Cardiovasc Imaging 5:433-440

11. Hong MK, Mintz GS, Lee CW et al (2004) Comparison of coronary plaque rupture between stable angina and acute myocardial infarction: a three-vessel intravascular ultrasound study in 235 patients. Circulation 110:928-933

12. Kataiwa H, Tanaka A, Kitabata $\mathrm{H}$ et al (2011) Head to head comparison between the conventional balloon occlusion method and the non-occlusion method for optical coherence tomography. Int J Cardiol 146:186-190

13. Imola F, Mallus MT, Ramazzotti V et al (2010) Safety and feasibility of frequency domain optical coherence tomography to guide decision making in percutaneous coronary intervention. EuroIntervention $\mathrm{J}$ EuroPCR Collab Work Group on Interv Cardiol Eur Soc Cardiol 6:575-581

14. Yamaguchi T, Terashima M, Akasaka T et al (2008) Safety and feasibility of an intravascular optical coherence tomography image wire system in the clinical setting. Am J Cardiol 101:562-567
15. Guedes A, Keller PF, L'Allier PL et al (2005) Long-term safety of intravascular ultrasound in nontransplant, nonintervened, atherosclerotic coronary arteries. J Am Coll Cardiol 45:559-564

16. Hausmann D, Erbel R, Alibelli-Chemarin MJ et al (1995) The safety of intracoronary ultrasound. A multicenter survey of 2207 examinations. Circulation 91:623-630

17. Van der Giessen W (2010) Intracoronary device insertion induces temporary, but stents induce chronic endothelial damage. Abstract Presented at ESC

18. Burke AP, Kolodgie FD, Farb A et al (2001) Healed plaque ruptures and sudden coronary death: evidence that subclinical rupture has a role in plaque progression. Circulation 103:934-940

19. Pinto DS, Stone GW, Ellis SG, Cox DA et al (2006) Impact of routine angiographic follow-up on the clinical benefits of paclitaxel -eluting stents: results from the TAXUS-IV trial. J Am Coll Cardiol 48:32-36 